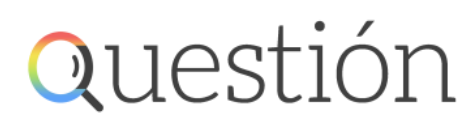

Periodismo / Comunicación ISSN 1669-6581
- Av. $44 \mathrm{~N}^{\circ} 676,1^{\circ}$ piso

CP 1900 - La Plata - Argentina

www.perio.unlp.edu.ar/question

Matrices de pensamiento audiovisual para pensar la comunicación en tiempos de Pandemia: análisis y prospectiva del rol de los medios de comunicación

Luis Barreras - Leonardo Murolo - Fernando Irigaray - Alejandra García Vargas - Pedro Arturo Gómez - Mariana Yepes https://doi.org/10.24215/16696581e356

\title{
Matrices de pensamiento audiovisual para pensar la comunicación en tiempos de Pandemia: Análisis y prospectiva del rol de los medios de comunicación
}

\section{Audiovisual thought matrices to think about communication in times of Pandemic: Analysis and prospective of the role of the media}

\begin{abstract}
Luis Barreras
Es licenciado en Comunicación Social. Secretario del Departamento de Ciencias Sociales de la UNDAV. Profesor e investigador de las Universidades Nacionales de Avellaneda, La Pampa y La Plata. Investigador Categoría II. Ha publicado libros, capítulos de libros y artículos en revistas haciendo foco en temas directamente relacionados a su especialidad: Análisis y Crítica medios. lbarreras@undav.edu.ar

Leonardo Murolo Es doctor en Comunicación Social. Director de la Licenciatura en Comunicación Social de la Universidad Nacional de Quilmes. Profesor e Investigador de la Universidades Nacionales de La Plata y Quilmes en grado y posgrado. Ha publicado Libros, Capítulos de libros y artículos en revistas con temas relacionados al campo de la comunicación digital audiovisual. nlmurolo@unq.edu.ar http://orcid.org/0000-0002-0987-5853
\end{abstract}

Fernando Irigaray

Es Magister en Nuevas Tecnologías de la Información y de la Comunicación (UNED - España), Licenciado en Comunicación Social en la Universidad Nacional de Rosario (UNR). Director de la Maestría / Especialización en Comunicación Digital Interactiva y de Comunicación Multimedial en la UNR. Dirige y produce programas y documentales para TV, interactivos y transmedia. Obtuvo el Premio Internacional Rey de España Categoría Periodismo

Digital 2013. Es profesor e investigador de la UNR y ha realizado diversas publicaciones sobre su especialidad las narrativas transmedia. fgirigaray@gmail.com

Alejandra García Vargas Es Doctora en Comunicación por la Universidad Nacional de Córdoba. Docente-investigadora a cargo de cátedras y proyectos de investigación en las Universidades Nacionales de Jujuy y de Salta. Codirectora del CESDE (Centro de estudios Socioeconómicos para el Desarrollo con Equidad, FCE, UNJu). Investigadora categoría II alegarciavargas@gmail.com

Pedro Arturo Gómez

Es Magister en Lingüística por El Colegio de México. Profesor en la Escuela de Cine, Video y Televisión de la UNT, en la carrera de Ciencias de la Comunicación de la UNT y la UCSE. También es escritor y crítico de cine. Ha realizado 
diversas publicaciones en libros, capítulos y escribió varios artículos sobre audiovisuales para publicaciones argentinas y del extranjero peargo50@gmail.com

Mariana Yepez Estudiante avanzada en Artes Audiovisuales Universidad Nacional Experimental de las Artes (UNEARTE-Venezuela). Productora para diversos proyectos estudiantiles y cinematográficos. Es productora de noticias de TELESUR. marianayepez94@gmail.com

\section{Palabras Clave}

Miradas - Diálogos - Medios de comunicación - Pandemia

\section{Key Words}

Looks - Dialogues - Media - Pandemic

Este texto fílmico se plantea como una matriz de pensamiento, desde diversas miradas de la comunicación y el periodismo, que promueve un diálogo de saberes sobre la problemática de la Pandemia del COVID-19. En ese sentido, la propuesta no implica un conocimiento acabado sino un punto de partida para analizar críticamente el rol de los medios de comunicación en sus diversos géneros y formatos.

En definitiva, implica pensar lo audiovisual como un campo de producción de conocimiento, en la medida en la que conocemos como el otro conoce y entendemos, en términos de Jesús Martín Barbero, que la comunicación es reconocimiento, es diálogo, es educación. Es en esa medida, que esperamos que este material sirva para pensar y repensar el rol de los medios de comunicación y nuestras propias prácticas ante la Pandemia.

\section{Enlace}

https://www.youtube.com/watch?v=Ekr1oZuOzwE

Nota: La producción de este corto, los escenarios, audios y videos son las condiciones de producción que cada integrante del mismo pudo adoptar en el contexto actual de aislamiento social, preventivo y obligatorio.

La edición fue realizada por el Lic. Luis Barreras. 